\title{
LA FEMME VOYAGEUSE DANS LES HÉROÏDES 10, 12 ET 21: LECTURES INTRA-OVIDIENNES
}

\author{
Par Stella Alekou
}

Summary: In this paper, the discussion centres on the portrayal of the female traveller in Ovid's Heroides 10,12 and 21. Notwithstanding the predominance of travelling narratives in the collection, most letters tend to focus on the description of the heroes' journeys as passively attested by the abandoned puellae, whereas the epistles of Ariadne, Medea and Cydippe explicitly address the issue of travelling as - also - a feminine activity. The examination of the travel motif will encourage us to review critically the wellestablished and quite restricted perception of women in Ovid's Heroides as mere elegiac puellae in love. The paper will tackle the recontextualisation of the travel metaphor from the text of two exiled women to Cydippe's final words - possibly written during Ovid's relegatio-, to examine the poet's plea for survival and commemoration.

Pour celui qui cherche à interpréter la représentation de la figure féminine chez Ovide, le recueil des Héroïdes demeure une source essentielle et inépuisable d'herméneutique. Nous examinerons dans les pages suivantes la nature que revêt la présentation de l'héroïne ovidienne, à partir des lettres qui n'ont pas fait le sujet d'une étude systématique jusqu'à présent. En effet, bien que le poète de Sulmone attribue à Médée tout comme à Cydippe le rôle de l'écrivain fictif, ${ }^{1}$ la moindre association en ce

1 La lettre 12 a suscité des études se focalisant sur la mise en question de son authenticité, comme, par exemple, Knox 1986: 207-23. Les arguments mentionnés, bien que stimulants, nous paraissent peu convaincants. Nous traiterons donc ce texte en tant que composition ovidienne, tout comme la lettre double d'Acontius et Cydippe. Sur l'authenticité des lettres doubles voir Burton 1908: 121-55. 
qui concerne l'illustration ovidienne des deux figures semblerait paradoxale: d'un côté, la princesse barbare, une des femmes mythologiques les plus discutées, est auto-décrite dans sa lettre pseudo-biographique comme meurtrière ; ${ }^{2}$ de l'autre, peu commentée dans la tradition littéraire, la jeune et innocente Cydippe se présente dans une paire épistolaire comme 'l'Autre' d'Acontius, étant, lors de son voyage à Délos, la victime d'une ruse malfaisante. ${ }^{3}$ Le choix d'étudier ces lettres paraît toutefois moins surprenant dès lors qu'elles sont comparées avec l'épitre d'Ariane, la femme abandonnée, ayant été endormie, sur une île déserte. Car le parallélisme entre les épîtres des deux femmes 'en exil', notamment Ariane et Médée, et la dernière lettre du recueil, celle de Cydippe souffrant d'une maladie grave, ouvre la voie vers une perspective que les spécialistes d'Ovide ont omis d'observer et qui mérite toute notre attention: celle de la femme voyageuse.

Plus exactement, le voyage permet le déplacement des héroïnes et favorise leur approchement, privilégié par des liens préexistants, géographiques ou bien généalogiques: la fuite d'Ariane, fille de Pasiphaé, à Naxos, la ville d'origine de Cydippe, introduit le lecteur à un rite de passage et la relie ainsi de surcroît à Médée, nièce de Pasiphaé, souvent étudiée comme sa 'Doppelgänger'. ${ }^{4}$ Notre lecture vise à traiter Cydippe

2 Sur cette illustration de Médée voir le travail de Michalopoulos 2004: 97-124.

3 La trame dramatique du mythe que traite la lettre double, entre Acontius et Cydippe, se constitue de manière générale de la façon suivante: un jeune homme de Céos, Acontius, voit pendant les fêtes de Diane et dans le temple de la déesse une fille de Délos, Cydippe, et lance une pomme à ses pieds, après avoir écrit la formule suivante de serment: 'Je jure par Artémis d'épouser Acontius'. La ramassant, la nourrice la donne à sa maîtresse, qui lit l'inscription à voix haute. Par conséquent, chaque fois que son père Céyx fait le nécessaire pour la préparation du mariage de sa fille et des prétendants autres qu'Acontius, elle est prise d'une fièvre violente. Ayant consulté l'oracle d'Apollon, Céyx obéit à la prescription du dieu et exécute la promesse de sa fille. Voir Call. Aet. 67-75. Nous soulignons également la version transmise par l'épistolier Aristaenète (1.10) dérivée de Callimaque. Le mythe en question est évoqué allusivement dans Verg. Ecl. 2, ainsi que plus explicitement dans Prop. 1.18.

4 Les légendes de Médée et d'Ariane mettent l'accent sur les instants du sacrifice et de la trahison, la promesse, la perfidie et l'abandon. Voir Dangel 2002:127-41. Voir aussi Fulkerson 2005: 35-36. Sur les thèmes élégiaques que les héroïnes du recueil partagent voir Landolfi 2000. Voir en particulier pp. 193 sqq. sur le sujet de l'abandon, pp. 204 sqq. sur le sujet de l'amour et p. 208 sur le sujet de l'attente. Sur les liens entre 
comme une lectrice érudite d'Ovide et donc une lectrice des lettres d'Ariane et de Médée. La problématique de notre travail réside dans le statut polyvalent que les trois textes accordent à la métaphore du voyage, comme lieu de transition entre l'art scriptural et l'art sculptural, dans une étude différenciée des artes memoriae. Les quatre axes principaux - le voyage, la mémoire, l'ecphrasis et la parole métapoétique et juridique - visent à mettre en relief un jeu polyphonique d'identifications et de distanciations, d'abord, entre Cydippe et les autres 'locutrices', ensuite, entre les 'épistolières' et leurs lecteurs, et, enfin, entre le poète et ses intertextes. Les lettres seront donc étudiées dans leur double fonction, à la fois comme œuvres statiques absorbant les qualités de l'ecphrasis et en mouvement, privilégiant la reprise créative du souvenir et l'originalité ingénieuse que revêt la parole-défense de la femme ovidienne.

\section{LE VOYAGE: À LA RECHERCHE DES CAUSES}

Le déplacement est une activité-clé dans les trois légendes ${ }^{5}$, mais surtout dans celle de la fille d'Æétès, constituée d'une succession de crimes et ponctuée d'un nombre considérable de fuites. Nous rappelons que de Colchide, où, amoureuse de Jason, Médée aide les Argonautes à accomplir 'les tâches impossibles' imposées par son père, la princesse barbare met en œuvre une première fuite pendant laquelle elle tue son frère; à Iolcos elle met fin à la vie de Pélias et ensuite à Corinthe, où elle est réfugiée avec son époux, elle tue sa rivale et ses enfants. La lettre 12 s'arrête à Corinthe, lors du deuxième mariage de Jason (12.137 sqq.) et avant la réalisation des meurtres de Créuse et des enfants de Médée. L'épître ne traite donc pas les évènements déroulés à Athènes où l'héroïne s'enfuit

l'illustration de Médée chez Apollonios de Rhodes et Euripide et celle d'Ariane de Catulle voir Armstrong 2006: 204-12; Clare 1996: 75; DeBrohun 1999: 427-29 ainsi que Theodorakopoulos 2000:121-29, qui se réfère également à la tragédie d'Ennius, Medea Exul.

5 Sur la métaphore du voyage, présente dans presque toutes les lettres, voir Jouteur 2007: 93-120. 
encore, pour retourner, à la fin, à sa ville natale; le lecteur est appelé à tracer le voyage intertextuel de l'epistula afin de combler les lacunes. ${ }^{6}$

L'introduction in medias res de la lettre 12 est marquée par l'arrivée du grand héros des Argonautes en Colchide (12.7-11: Ei mihi! cur umquam iuvenalibus acta lacertis / Phrixeam petiit Pelias arbor ovem ? / Cur umquam Colchi Magnetida vidimus Argon / Turbaque Phasiacam Graia bibistis aquam? / Cur mihi plus aequo flavi placuere capilli;). ${ }^{7}$ Bien que cette introduction semble à première vue définir ses liens intertextuels avec les textes fondateurs, ${ }^{8}$ la lettre dépasse les limites 'médéocentriques' de son histoire, lorsque son auteur fictif mène subtilement le récit du périple vers la reconfiguration du voyage traité dans un autre texte. La présentation du voyage Argonautique dans la narration de Médée renvoie explicitement à l'ouverture du Carmen 64 de Catulle, qui traite ce même sujet afin d'introduire au lecteur le mythe de Thétis et Pélée (64.1-13). ${ }^{9}$

L'illustration de l'arrivée de l'Argo dans le territoire barbare où règne Æétès fait allusion au texte catullien en écho inversé, puisqu'elle se focalise moins sur une union, celle de Thétis et Pélée, et plus sur une séparation, illustrée par un départ de Naxos et présenté dans le Carmen 64 sous la forme d'une ecphrasis: celui de la nef de Thésée, tissé sur le couvre-lit des mariés. Le compte est repris dans l'épitre ovidienne d'Ariane (10.3-4: Quae legis, ex illo, Theseu, tibi litore mitto, / Vnde tuam sine me vela tulere ratem). Située dans la perspective du voyage, qui conduit à une expérience et une aventure non-ordinaires, la lettre d'Ariane, qui aide Thésée, meurtrier de son demi-frère, le Minotaure, à s'échapper du Labyrinthe, s'arrête, elle aussi, lorsque son 'épistolière' se trouve, tout comme Médée, trahie et abandonnée (10.5-6: In quo me somnusque meus male prodidit et $\mathrm{tu}$, / Per facinus somnis insidiate meis). La description des deux voyages est

6 Nous notons les œuvres d'Ennius, Medea Exul, d'Accius, Medea sive Argonautae, ainsi que les œuvres de Pacuvius (Médus), de Sénèque (Médée) et de Valérius Flaccus (Argonautiques). Voir aussi Cic. Cael.; Pi. P. 4; Ap. Rhod. Argon.; E. Med.

7 Pour le texte et la traduction nous avons utilisé l'édition de Bornecque 2005.

8 Voir les introductions du mythe chez Ennius, Medea Exul 103, 208-16 et chez Accius, Medea sive Argonautae 1.467-78. Cf. E. Med. 1-8 et Ap. Rhod. Argon. 2.169-74 et 549-54.

9 Voir Dufallo 2012: 41. 
marquée par le besoin des 'épistolières' de définir les causes de leur malheur - et de leur exil (10.66: exul; 12.110: in exilio) ${ }^{10}$ - : chez Médée, des questions rhétoriques, introduites par cur à trois reprises, abordent le sujet de son regret $(12.7,9,11)$; chez Ariane, la triple réitération du terme crudelis met l'accent sur la personnification des somnus, ventus et dextera, présentés comme la cause de son abandon à l'île de Naxos (10.111-116: Crudeles somni, quid me tenuistis inertem? / Ah! semel aeterna nocte premenda fui. / Vos quoque crudeles venti, nimiumque parati / Flaminaque in lacrimas officiosa meas; / Dextera crudelis, quae me fratremque necavit, / Et data poscenti, nomen inane, fides).

Naxos, ville natale de la dernière des héroïnes ovidiennes, ainsi que lieu de départ et destination finale, accueille les lamentations de deux figures face à la mort: Ariane abandonnée et Cydippe souffrant d'une maladie grave. La maladie de Cydippe est directement liée à la lecture involontaire: ayant juré à son insu d'épouser Acontius, elle est prise d'une fièvre lors des préparatifs de son mariage avec un autre homme, alors qu'Acontius ne peut surmonter sa passion et fuit dans la solitude des forêts, gravant le nom de sa bien-aimée sur l'écorce des arbres. Le lecteur ovidien connaît la source principale du mythe, notamment les Aitia de Callimaque, qui traitent les causes des symptômes, dans une démystification de la 'maladie sacrée' (3.75.11-19). Ovide reprend le mythe au moment où, malade et ayant reçu une lettre de la part d'Acontius, l'héroïne compose -à l'exemple d'Ariane - à Naxos elle aussi, un texte-réponse qui la conduit ainsi à la recherche des causes de son état de santé. L'interrogation portant sur son voyage de Naxos à Délos reprend la formule du trio des lettres 10 et 12, par la répétition du nom pede, terme-indice du déplacement (21.71-72: Quo pede processi? quo me pede limine movi? / Picta citae tetigi quo pede texta ratis?). ${ }^{11}$ L'image des pedes, sur lesquels la pomme fut jetée (21.109: Mittitur ante pedes malum cum carmine tali), lie subtilement à la métaphore du voyage le mouvement accompli dans l'envoi de

10 Cf. Ov. Her. 7.115. Didon se présente dans le recueil en tant que femme exilée, tout comme Médée et Ariane; toutefois, son épître s'achève lors de son suicide, annoncé et décrit aux derniers vers. Contrairement donc aux 'locutrices' des Lettres 10 et 12, la fin de sa lettre limite son statut de femme voyageuse dans le passé.

11 Cf. Ov. Her. 21.98: Erramusque vago per loca sacra pede. 
la lettre et de la pomme porteuse d'une inscription ${ }^{12}$. Ariane se sert de la même métonymie ${ }^{13}$, lorsque le sable piégeant ses pieds de jeune fille la retarde dans son effort pour courir, faisant ainsi allusion non plus au mouvement, mais à la nature statique du texte (10.19-20: Nunc huc, nunc illuc, et utroque sine ordine, curro; / Alta puellares tardat harena pedes). ${ }^{14}$ Son illustration à l'heure de la rédaction de la lettre se trouve en opposition avec l'emploi du terme mitto au début de son texte (10.1), qui renvoie

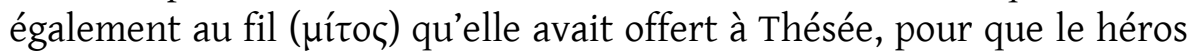
sorte sauf et vainqueur du labyrinthe (10.103-4: Cum tibi, ne victor tecto morerere recurvo, / Quae regerent passus, pro duce fila dedi). ${ }^{15}$

Cydippe va encore plus loin dans la subversion de sa reprise: en réponse à l'allégation de l'héroïne 10, selon laquelle les sommeils et les vents cruels (10.111: crudeles somni, 10.113: crudeles venti) ont provoqué sa souffrance, la dernière des Héroïnes précise que 'c'est folie d'accuser l'inconstance des vents' (21.78: Sed stultum est venti de levitate queri) ${ }^{16}$, et que le sommeil, le meilleur prétexte d'une longue solitude, cesse d'être crédible (21.23-24: Mox, ubi, secreti longi causa optima, somnus / Credibilis tarda desinit esse mora). Invitant le lecteur à une première mise en question des causes (10.111-116; 12.7-11), elle l'encourage à se focaliser sur la distanciation ovidienne vis à vis du texte source ${ }^{17}$ mais aussi des autres lettres. Ainsi, Cydippe ajoute encore que la cause est cachée (21.55: causa latet) et se focalise non plus sur le 'pourquoi' que traitent les héroïnes 10 et 12 , mais sur le 'comment' (21.71-72: quo).

12 Sur la métaphore du voyage voir Höschele 2007: 333.

13 Sur la métonymie dans les Héroïdes voir Casanova-Robin 2007: 60.

14 L'état statique fait aussi allusion à l'illustration d'Ariane enfermée dans une ecphrasis chez Catulle (carm. 64.50 sqq.).

15 Voir Michalopoulos 2002: 95-97 sur le jeu de mots en question. Le verbe mitto est principal dans la construction des héroïnes et des héros en voyage. Cf. Ov. Her. 1.1, 1.36, 1.64-65, 2.133, 3.64, 3.127, 4.2, 4.37, 5.2 , 6.8, 7.72, 9.1, 10.4, 11.2, 11.95, 13.1, 14.1, $15.168,15.185$.

16 Voir Jouteur 2007: 2 sur les variantes autour du vent et la loi de répétition dans le recueil.

17 L'accent mis sur les causae, un terme clé dans la lettre d'Acontius, apparaissant 11 fois, dévoile, dans ce contexte en particulier, les rapports de la version ovidienne avec l'œuvre-source, notamment les Aitia de Callimaque. Voir sur ce rapport Michalopoulos 2014: 263. 
L'interrogation sur les moyens et les raisons des causalités dans les narrations d'Ariane, Médée et Cydippe nous permet donc de les observer comme une interférence de plusieurs points de vue..$^{18}$ De caractère complémentaire, le récit du voyage fait naître une écriture qui n'est qu'une composition de plusieurs aperçus partiaux et sélectifs du souvenir, lesquels, une fois 'revus' ensemble, forment un tout cohérent et mémorable. ${ }^{19}$

\section{L'ART DE REVOIR ET L'ART DE MÉMOIRE}

La complémentarité des trois textes que l'on examine réside dans la construction de l'image d'un voyage, souvenu et décrit en train de se réaliser, lors de ses deux phases, l'accent étant mis sur un départ (lettre 10), sur un itinéraire (lettre 21) et une arrivée (lettre 12). Dans les pages suivantes, nous montrerons que ce que présentent ces textes concerne moins l'esquisse du monde extérieur - notamment le paysage ${ }^{20}$ - que des figures féminines, décrites lorsqu'elles sont en train d'apercevoir. ${ }^{21}$ Trois

18 Sur l'interférence de plusieurs points de vue dans la narration voir Bordas 2010: 20.

19 Spentzou 2003: 28 remarque que les modes narratifs dans les Héroïdes permettent à plusieurs destinataires, à différents moments dans le temps, de se livrer à des lectures imprévisibles et transgressives. Cydippe est la seule des Héroïdes à ne pas avoir employé les termes epistula et littera pour sa lettre. Scriptum paraît plus apte à décrire la multitude des textes qui y surgissent, selon Michalopoulos 2014: 257.

20 Jouteur 2007: 120 mentionne que "[s]i la mer, si le vent sont si changeants, c'est que leurs multiples variations ne font que refléter le remous des pensées et des sentiments dans un rapport analogique intime entre l'intérieur et l'extérieur. La poésie, soumise à l'empire de la rhétorique, quitte la sphère référentielle pour s'afficher comme autotélique." La critique ajoute : "Le véritable paysage des Héroïdes est intérieur. Ce paysage est celui du champ infini de l'attente, qui suscite la parole 'inépuisable'." Voir aussi Tola 2005: 66 sur l'image de l'eau dans les Héroïdes.

21 Ariane est à la fois protagoniste et spectatrice de son histoire; elle devient l'objet de son propre regard, selon Vaiopoulos 2017: 33. Voir aussi Goldhill 2007: 2: "There is a highly developed discourse of viewing in Hellenistic culture, for which the notion of phantasia - impression - is crucial. It offers a philosophical and physiological explanation of how viewing functions, and is related to psychological processes and the production of speech. This demands that ekphrasis also be related to the idea of spectacle, displaying in art galleries, museums, rhetorical performance." 
perspectives attirent le regard du lecteur: le paysage comme objet de focalisation, le personnage, tantôt objet décrit, tantôt spectateur actif, et le texte, à la fois véhicule d'accès à l'image souvenue et image mémorable en soi.

La mémoire est un terme clé chez les trois héroïnes ${ }^{22}$ : Médée commence son œuvre pseudo-biographique déclarant qu'elle s'en souvient (12.1: memini) - en se référant à son aide offerte à Jason -, alors qu'Ariane exprime le besoin de ne plus être oubliée par Thésée, l'oubli ayant suscité l'écriture de sa lettre (10.129-30: Me quoque narrato sola in tellure relictam. / Non ego sum titulis subripienda tuis). Paradoxalement, Cydippe met l'accent sur son amnésie intentionnelle et sa mémoire volontairement courte, précisant qu'elle ne se rappelle rien, et qu'elle ne veut nullement rapporter tout ce qu'elle y a vu (21.103-4: Neque enim meminive libetve / Quidquid ibi vidi dicere). Elle offre donc un regard distancié non seulement sur son passé - aussi intertextuel -, mais également sur les héroïnes qui l'ont précédée, ainsi que sur leurs souvenirs. Cette posture privilégie une lecture interdépendante des trois textes, puisque l'écriture de la dernière lettre se trouve liée à la réception de plusieurs textes: de l'inscription sur la pomme, mais aussi de la lettre d'Ariane et celle de Médée, ainsi que de l'intertexte ovidien.

En décrivant son voyage à Délos, Cydippe met l'accent sur les statues qu'elle admire sur l'autel ainsi que sur le lieu sacré qui l'entoure, dans un intéressant mélange d'éléments de nature et de créations artistiques (21.100-2: Miror et in cunctis stantia signa locis. / Miror et innumeris structam de cornibus aram. / Et de qua pariens arbore nixa dea est,). Sa double focalisation est mise en évidence par le verbe miror à deux reprises (21.100-1). De manière peu différente, Médée et Ariane se placent dans le passé afin de décrire leur perception visuelle de l'être aimé, par l'emploi du verbe vidi, de nouveau à deux reprises (12.31: Tunc ego te vidi; tunc coepi scire quis esses; 12.33 Et vidi et perii nec notis ignibus arsi; 10. 29-32: Inde ego (nam ventis quoque sum crudelibus usa) / Vidi praecipiti carbasa tenta Noto / Aut vidi aut

22 Sur la mémoire dans les Héroïdes voir Stroh 1991: 201-44. Cf. Jouteur 2007: 2 sur la mémoire visuelle et Davis 2012: 33-48 sur la mémoire en tant que note alexandrine. Voir aussi Conte 1986: 23-24 sur la mémoire et l'allusion. Sur l'emploi de la notion de mémoire dans les Héroïdes voir Ov. Her. 3.101, 5.23-24, 6.64, 8.75, 10.92, 12.1, 15.43. 
acie tamquam [quae me] vidisse putarem / Frigidior glacie semianimisque fui). ${ }^{23}$ La présence dédoublée du verbe dans la lettre 10, ne concernant plus une première, mais une dernière rencontre avec son amant, décrit ce que la jeune femme voit (10.30), ou ce que son regard croit voir (10.31: vidisse putarem); il est ainsi insinué que l'image peut causer la perte d'objectivité, l'aperçu étant propre à perturber le sujet de focalisation, comme dans le cas de Médée (12.33: et vidi et perii). L'étude parallèle du souvenir, en ce qui concerne le déplacement de Cydippe de Naxos à Délos, l'arrivée de Jason à Colchide et le départ de Thésée de Naxos, permet donc la mise en évidence d'un jeu de dédoublement et dévoile ainsi une double transmission de l'image: ce que le lecteur voit à travers le texte et connaît sur l'histoire, et ce que l'héroïne enregistre avec ses yeux s'entrecroisent à partir d'une chaine lexicale de rapports souvent conflictuels.

Le conflit entre le réel, ce que l'on voit donc par les yeux, et le fictif, qui renvoie à ce qu'on croit connaître ou que l'on construit par l'esprit, reflété dans les doubles points de vue qui semblent subjectifs (vidi, miror), est également ressenti dans les effets indésirables de la vue, qui varient, eux aussi: l'image du feu dans le cas de Médée (12.33-34: Et uidi et perii nec notis ignibus arsi, / Ardet ut ad magnos pinea taeda deos) et de la glace dans le cas d'Ariane (10.49: Aut mare prospiciens in saxo frigida sedi,) s'allient dans l'illustration de Cydippe, dont le visage, à partir du moment où elle voit l'inscription sur le fruit, crée par son ton un intéressant mélange de rouge et de blanc, renvoyant ainsi, d'un côté, aux teintes de la pomme, et, de l'autre, aux nuances des statues (21.217-22: Concidimus macie; color est sine sanguine, qualem / In pomo refero mente fuisse tuo. / Candida nec mix to sublucent ora rubore. / Forma noui talis marmoris esse solet; / Argenti color est inter conuiuia talis, / Quod tactum gelidae frigore pallet aquae). ${ }^{24}$ La lecture et la réception d'un texte malfaisant produisent alors des effets comparables à ceux de l'expérience de la première et dernière rencontre, une dynamique d'interaction qui permet au discours polyphonique de l'héroïne ovidienne de progresser. ${ }^{25}$ Mais son effet réitéré dévoile d'abord

23 La symétrie entre les deux passages, qui prennent approximativement la même place dans les deux textes, est remarquable.

24 Voir Casanova-Robin 2008: 74.

25 Sur les effets désastreux de la vue pendant la première rencontre entre Médée et Jason dans la lettre 12 voir Aresi 2017:31-36. 
une posture cognitive: pour mieux comprendre sa place, Cydippe prend celle d'Ariane et de Médée, faisant ainsi retour sur la sienne et, en même temps, faisant émerger un point de vue qui semble être partagé, revu et établi comme 'vrai'. Comme le précise l'héroïne de la lettre 21, si les causes du malheur sont cachées, les maux sont bien visibles (21.55: mala nostra patent).

Bien que l'illustration de la figure féminine soit matérielle, l'absence de l'être aimé dicte la mise en œuvre d'illusions. Adonnées à la recherche d'objets symboliques apparaissant comme des métonymes de l'amant disparu, ${ }^{26}$ Ariane et Médée font agir les simulacres, par la répétition de mots dégageant une image constante. Le terme simulacrum est employé dans la lettre 10 pour décrire les simulacres des dieux préoccupant l'esprit d'Ariane, en suscitant en elle la peur (10.95: timeo simulacra deorum), ${ }^{27}$ alors que, dans la lettre 12 , définie de manière quasi étymologique, la similitude de l'image entre père et fils (12.189: Et nimium similes tibi sunt et imagine tangor) génère le simulacre qui bouleverse Médée (12.190: lumina madent). Finalement, non plus au sens figuré, la double lettre d'Acontius et Cydippe met l'accent sur le simulacre de la pomme porteuse d'une nouvelle inscription, terrifiante pour Cydippe (20.239-42: Aurea ponetur mali felicis imago, / Causaque versiculis scripta duobus erit: / 'Effigie pomi testatur Acontius huius / Quae fuerint in eo scripta, fuisse rata.'). Faisant partie d'une paire, sa lettre apparait comme un texte dédoublé en soi, une note inachevée qui renvoie le lecteur en arrière. ${ }^{28}$ La nature autoréférentielle du dernier texte ovidien, qui met en relief le procédé de son écriture et de sa lecture, témoignant de la crainte de l'épistolière' de se répéter, est exprimée dès le début de l'épître (21.3-4: ... Pertimui scriptumque tuum sine murmure legi, / Iuraret ne quos inscia lingua deos). Celle-ci reflète, de surcroît, l'inquiétude de l'artiste effectuant une introspection subjective sur l'originalité d'une œuvre qui semble contenir des histoires similaires, ${ }^{29}$

26 Casanova-Robin 2008: 85. En ce qui concerne la présence et l'absence dans le cadre épistolaire des Héroïdes voir Hardie 2002: 106-42; Lindheim 2003: 20-22; Jolivet 2001: 233.

27 Voir Volk 2003: 349 et Knox 1995: 250 sur les simulacres dans la lettre d'Ariane.

28 Rimell 2006: 171.

29 Sur la signification de la répétition dans les lettres doubles voir Rimell 2006: 158 et Jouteur 2007:2, qui note que les mots "se répondent et s'entrecroisent pour ressasser un signifié identique ou proche”. Cf. Michel 1994²: 65. 
ou bien des histoires déjà traitées dans d'autres textes. La mise en relief des effets de la vue fait ainsi avancer la réflexion du lecteur sur les sentiments que procure l'image des doubles. Le procès d'imitation est aussi abordé lorsque l'auteur insiste tout particulièrement sur la polysémie du terme simulacrum, un terme qui dessine également 'la statue', ${ }^{30}$ dans un essai de dissociation entre le texte et l'intertexte, ou bien entre le 'vrai' et le vraisemblable.

\section{L'ECPHRASIS: À LA RECHERCHE DU 'MOI' ET DE L' 'AUTRE'}

L'interaction de Cydippe avec les figures en marbre (21.101-2) renvoie au texte de Callimaque, qui se réfère explicitement à la vénération d'une statue qui illustre Ariane à Naxos, dans son traitement du mythe d'Acon-

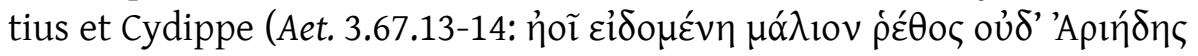

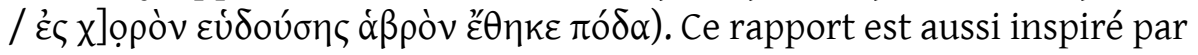
la lettre ovidienne d'Acontius, qui emploie dans sa description de la beauté de Cydippe des termes renvoyant à une figure en marbre (20.5762: Tu facis hoc oculique tui, quibus ignea cedunt / Sidera, qui flammae causa fuere meae; / Hoc faciunt flavi crines et eburnea cervix, / Quaeque, precor, veniant in mea colla manus, /Et decor et motus sine rusticitate pudentes, / Et, Thetidis qualis vix rear esse, pedes). Le moment et le lieu ne sont pas choisis au hasard: Acontius parle de la jeune femme de cette manière à Naxos, pendant qu'elle admire les statues dans le temple de Diane. Naxos est formée de roches métamorphiques et est célèbre dans l'Antiquité grâce à son marbre, ${ }^{31}$ un élément géologique qui privilégie la métamorphose rhéto-

30 OLD s.v. simulacrum 3. Voir Barchiesi 2001: 23, sur une triple interprétation du terme.

31 Voir sur Naxos par rapport au mythe d'Ariane le travail de Vaiopoulos 2017: 27-43. Sur l'illustration d'Ariane par rapport aux arts visuels voir Vaiopoulos 2017: 27. Cf. Verducci 1985: 244. Selon Beye 1982: 43-44, “[i]n the original story, the Argo is sailing out of the known world into the stream of the ocean which surrounds the world. It is sailing beyond the physical world, bounded by time, into the spirit world, and the Clashing Rocks are the point of entry. Pindar says (4.210-11) that the voyage of the Argonauts brought teleute ('death') to the rocks; they never moved again. That teleute is, it is argued, some kind of initiation, a rite de passage. The voyage through the rocks 
rique de la figure en ecphrasis sculpturale, déjà mise en œuvre chez Catulle (64.60-1: Quem procul ex alga maestis Minois ocellis, / Saxea ut effigies bacchantis, prospicit, eheu!). ${ }^{32}$

À l'exemple de l'illustration de la femme catullienne, chez Ovide, la reconstruction de la figure féminine adopte elle aussi les qualités des statues, d'abord dans la lettre d'Ariane, et devient ainsi le simulacre d'une œuvre de sculpture. Oubliée et délaissée, sur un roc (10.49: in saxo) ou 'en tant que roc elle-même' (10.50: Quamque lapis sedes, tam lapis ipsa fui), la princesse ovidienne dirige le regard du lecteur / spectateur vers son intertexte: en s'identifiant en tant que l' 'autre' de la Bacchante catullienne (10.48-49: Qualis ab Ogygio concita Baccha deo / Aut mare prospiciens in saxo frigida sedi,), elle rappelle qu'elle fait partie d'un projet plus grand, dans un autre contexte - celui de la Bacchante illustrée sur la broderie, offerte comme cadeau au mariage de Thétis et Pélée (64.61: Saxea ut effigies bacchantis, prospicit, eheu!). ${ }^{33}$ Mais la réitération du terme lapis (10.50), lorsque l'héroïne devient pierre en soi, absorbant les traits du matériel sculptural, nous conduit à apercevoir son corps à travers son fonctionnement artistique, dans une transmutation créative de l'élément de la nature en art. $^{34}$ En effet, face à l'abandon, la figure, soigneusement travaillée, devient une expression artistique qui traduit sa 'solitude endeuillée', dans une 'sublimation picturale'.35

Enfin, une autre princesse, Médée de Colchide, spectatrice forcée de l'abandon et témoin passif d'une rupture violente, participe, elle aussi involontairement, à la cérémonie matrimoniale entre son époux et la fille de Créon (12.137-39: Vt subito nostras Hymen cantatus ad aures / Venit et accenso lampades igne micant / Tibiaque effundit socialia carmina vobis).

is a teleute for the Argonauts as well, an ordeal, and thereafter they move to a new sphere". Voir aussi Lindsay 1965: 10-11. Cf. Ap. Rhod. Argon. 2.553-54.

32 Sur l'originalité de cette ecphrasis voir Laird 1993: 20. Sur le rapport entre le regard et l'ecphrasis catullienne voir Elsner 2007: 20.

33 Sur le symbolisme du roc voir Knox 1998: 72-83. Sur les connotations élégiaques du terme frigida voir Bolton 2009: 285 n. 29, qui le présente comme topos ovidien. Cf. Ov. Ars am. 2.1.5, 2.7.9, 3.5.42; Ov. Rem. 492; Ov. Her. 1.7, 2.123, 10.49, 11.117, 14.38, 14.40, $18.116,19.69$.

34 Cf. Ov. Her. 21.217-22.

35 Voir Casanova-Robin 2005: 126. Sur la représentation artistique d'Ariane dans l'art gréco-romain voir McNally 1985: 152-55. 
'Hors scène', cachée à l'intérieur de sa demeure, elle réagit au témoignage de son fils, qui décrit naïvement ce qu'il voit (12.151-52: 'Huc mihi, mater, adi! pompam pater, inquit, Iason / Ducit et adiunctos aureus urget equos.'). Bien que l'accent semble à première vue être mis sur la cérémonie lumineuse du mariage ${ }^{36}$ le lecteur est vite emporté par la violence de l'image de la femme en coulisses: les vêtements déchirés, le visage arraché, Médée échevelée imite la pétrification de la statue en lamentatio et renvoie ainsi à la figure en pierre de la lettre 10 (12.153-58: Protinus abscissa planxi mea pectora veste / Tuta nec a digitis ora fuere meis. / Ire animus mediae suadebat in agmina turbae / Sertaque compositis demere rapta comis; / Vix me continui quin sic laniata capillos / Clamarem 'meus est' iniceremque manus). L'image acoustique et visuelle de la cérémonie devient secondaire face à celle, non visible et sans son, de Médée; l'héroïne s'éloigne ainsi de son illustration traditionnelle de pauvre, barbare et coupable qu'elle attribue à son lecteur fictif ou bien réel (12.160: Nunc tibi sum pauper, nunc tibi visa nocens) et demande, par écrit, d'être vue différemment.

Dans un autre cadre mythologique, Cydippe devient aussi objet de focalisation (21.223: Si me nunc videas, visam prius esse negabis), ${ }^{37}$ en se situant dans un contexte peu différent, celui donc d'un mariage auquel elle assiste, tout comme Médée, contre sa propre volonté, lors du rituel à l'heure des cérémonies religieuses, traduisant le processus de sa propre toilette nuptiale (21.89-92: Quos idem solitos postquam revocavit ad ortus, / Comuntur nostrae matre iubente comae; / Ipsa dedit gemmas digitis et crinibus aurum / Et vestes umeris induit ipsa meis). ${ }^{38}$ L'écho dans la description de l'héroïne 21 (21.223: nunc ... visam), renvoyant plus aux funérailles qu'au mariage, avec le texte de Médée (12.106: nunc ... visa), qui enregistre sa réception personnelle des chants nuptiaux comme funéraires (12.140-41: Ei mihi! funerea flebiliora tuba, / Pertimui nec adhuc tantum scelus esse putabam;), ${ }^{39}$ se confirme aussi par la focalisation des deux 'épistolières' sur

36 Jouteur 2009: 77-78: “C'est la musique de la fête qui informe de l'événement, avec un contraste ironique, entre la cacophonie joyeuse de la foule et la contrition frustrée de l'héroïne."

37 Cf. Ov. Her. 21.106: Si, tibi deformis, quod mallem, visa fuissem.

38 Voir Casanova-Robin 2008: 80.

39 Cette illustration du mariage, qui fait allusion à la mort de Créuse, symbolise surtout la fin du mariage de Médée avec Jason, selon la loi romaine. Sur les conditions en ce 
leur 'moi dé/construit' (12.153-58 et 21.90-92). Contrairement à l'autodescription de Médée, la coiffure de la jeune fille est bien arrangée, ses doigts se présentent comme subtilement décorés et le vêtement paraît couvrant ses épaules. L'inversion de l'image est mise au surplus en relief par l'inversion de l'ordre des mots (veste, digitis et capillos dans la lettre 12 et comae, digitis, vestes dans la lettre 21 ).

L'accent mis sur le tissu couvrant le corps féminin confirme le rapport du texte ovidien avec sa source, notamment le Carmen 64, et plus exactement le couvre-lit du mariage de Thétis (64.47-51: Pulvinar vero divae geniale locatur / Sedibus in mediis, Indo quod dente politum / Tincta tegit roseo conchyli purpura fuco. / Haec vestis priscis hominum variata figuris / Herorum mira virtutes indicat arte), mais aussi avec le voile entourant la figure à demi nue, esquissée dans une ecphrasis (64.63-67: Non flavo retinens subtilem vertice mitram, / Non contecta levi nudatum pectus amictu. / Non tereti strophio lactentis vincta papillas, / Omnia quae toto delapsa e corpore passim / Ipsius ante pedes fluctus salis adludebant). ${ }^{40}$ La valeur symbolique du tissu, ${ }^{41}$ qui associe en opposition l'histoire de mariage de Thétis à celle de la séparation d'Ariane, ${ }^{42}$ suggère un rite de passage d'un contexte mythologique à un autre et, paradoxalement, d'une union à une séparation. Le caractère ritualiste du voile dans la perspective d'un mariage ou des funérailles ${ }^{43}$ permet surtout à Ovide d'éloigner son épistolière fictive de son intertexte catullien, dont l'esthétique du dénudement semble être

qui concerne la validité du mariage romain voir Cic. de Orat. 1.40.183: Si iudicaretur certis quibusdam verbis, non novis nuptiis fieri cum superiore divortium.

40 La mise en abyme d'une deuxième ecphrasis concerne la statue d'une Bacchante alors qu'une troisième ecphrasis concerne les vêtements de la jeune femme (64.63-65); le tissu se trouve, grâce à sa finesse ainsi qu'à son mouvement équilibré et rythmique, en dialogue avec les flots de la mer et le corps à demi nu (64.68 sqq.). Cette mimesis ingénieuse du tissage, 'composée' sur le texte démontre la puissance de la poésie confrontée à un art d'imitation par l'image. Voir Barbaud 2006: 56.

41 Voir Scheid \& Svenbro 1994: 97-98 sur ce sujet.

42 Sur la question de composition dans le Carmen 64, et en particulier sur le plan général du poème, voir Waltz 1945: 95-97 et Ramain 1922: 137. Cf. Armstrong 2006: 190; Deroux 1986: 247-58; Duban 1980: 777-78; Kinsey 1965: 911-12; Putnam 1972: 225-65; Warden 1998: 398-413.

43 Sur une interprétation ritualiste du voile voir Cairns 2002: 73-93. 
insinuée dans l'illustration de l'héroïne 12 (12.153: Protinus abscissa planxi mea pectora veste).

Le refus de l'épistolière' 10 de se débarrasser de son voile, qui, mouillé et lourd de larmes, se retient sur son corps en pierre (10.138: Et tunicas lacrimis sicut ab imbre gravis), devient source d'inspiration pour Cydippe (21.92: Et vestes umeris induit ipsa meis), qui incorpore dans son moi l'esthétique retracée dans l'image élégante de la description que l'on trouve dans la lettre 10: évoquant à la fois le 'réel' et l'imaginaire, dans une ecphrasis qui se rejoint avec la présentation des silhouettes statiques sculptées à la main, l'image quasi mystique de la figure que couvre le voile, qui ne renvoie plus à l'union, mais aux préparatifs conduisant la victime-offre en sacrifice à l'autel, ${ }^{44} \mathrm{~s}$ 'y éclaircit. Par l'intervention du souvenir intratextuel que favorise l'emploi du voile, Cydippe impose la présence maudite d'Ariane dans la construction de son portrait et voue ainsi à l'échec son union avec Acontius. Symbole de séparation dans la pratique de l'initiation, le voile permet donc à la dernière des héroïnes de mettre l'accent sur la fluidité qui existe entre les étapes de transition: entre l'union et la rupture se trouve la liminalité, dont l'ambiguïté et l'ambivalence relèvent le souci d'identité.

Par la triple reprise épistolaire de l'ecphrasis, le poète de Sulmone donne la parole en écriture à la figure callimaquéenne ${ }^{45}$ comme Catulle l'avait fait à une silhouette muette en marbre, ${ }^{46}$ et confère à la femme meurtrière une esthétisation qui ne devient évidente qu'à partir d'une lecture intra-ovidienne; Ovide favorise ainsi l'éloignement de Médée de son portrait traditionnel de femme criminelle. La distance que prend le poète à l'égard des histoires précédentes lui permet d'exploiter l'ecphrasis dans un large spectre, en donnant une dimension spectaculaire à ces héroïnes, dimension dont la reconstruction se situe entre illusion, allusion et réalisme.

45 Sur les différences entre les deux versions voir Michalopoulos 2014: 48-49.

46 Barbaud 2006: 57 et 62. 


\section{ACTIO RHÉTORIQUE ET VRAISEMBLANCE JURIDIQUE}

La polysémie et la polyphonie de l'image allusive sont mises au service de la défense ultime de l'héroïne ovidienne. Comme nous le montrerons dans les pages suivantes, Ariane, Médée et Cydippe abordent souvent le sujet du discours et révèlent fréquemment sa nature trompeuse, afin de défendre leur cause. Inscrite sur la pomme ou la lettre, prononcée dans les normes d'un contrat contraignant ou simplement sous la forme d'une promesse, la parole se manifeste en tant que stratagème rhétorique et art poétique qui vise à la victimisation des 'locutrices'.

C'est surtout Cydippe qui met l'accent sur le danger de la 'relecture', en réponse à la lettre d'Acontius qui, comme la nourrice dans le temple, lui ordonne de la lire jusqu'au bout (20.5 et 21.111: perlege) ${ }^{47}$ Elle explique que la lecture des paroles qui jurent ne devrait pas avoir l'effet d'un serment juré (21.145), d'une voix sans âme (21.143), et des mots dénués de leur force (21.144), et précise que celle qui jure c'est l'âme (21.137: Quae iurat, mens est). ${ }^{48}$ L'emploi du terme mens pour illustrer l'opposition entre la sphère spirituelle et la sphère physique apparaît bien avant dans les lettres d'Ariane et de Médée: vers la fin des épîtres, la princesse de Crète demande que Thésée l'aperçoive, non plus avec les yeux, mais avec l'esprit (10.135: non oculis, sed, qua potes, adspice mente) et la princesse de Colchis note que, bien que son cœur soit bouleversé, sa mens médite quelque chose de plus grand (12.212: Nescio quid certe mens mea maius agit).

À l'exemple d'Ariane, Médée juxtapose d'abord les oculi de Jason à ses lumina (12.36), faisant apparaître la dualité en conflit déjà présentée dans

47 Sur le dialogue des ouvertures des lettres 20 et 21 voir Michalopoulos 2014: 257, qui se focalise sur le sentiment de la peur, illustré dans les deux textes.

48 Cf. l'opposition entre le corps et l'esprit chez Titus Livius, 57-60. Selon Ducos 1984: 304 sqq. et 205, “[1]'opposition de la lettre et de l'esprit n'est assurément pas analysée clairement comme elle le sera au temps de Cicéron. [...] Et, à la fin de la République, juristes et écrivains n'ignoreront pas que certains actes peuvent respecter la loi dans sa forme sans le faire dans son esprit." La critique ajoute que la question la plus importante demeure celle de la controverse entre la lettre et l'esprit: scriptum et sententia; Cicéron s'y attache en nous montrant dans le De inventione comment on peut défendre l'une ou l'autre. 
la lettre 10; car oculus désigne les yeux en tant que partie du corps ${ }^{49}$ tandis que le terme lumina représente 'la compréhension', 'l'illumination mentale, 50 'les yeux actifs grâce auxquels on possède le pouvoir de la vision,.$^{51}$ De manière similaire, la dualité par rapport au sens de la vue réapparait en juxtaposition - aussi temporelle par le tunc (12.3) et le nunc (12.211) - entre l'art de mémoire, la capacité donc de revoir le passé (12.1: ars, memini), et le pouvoir divin de prévoir l'avenir (12.212: Viderit ista deus). Cette dualité est appuyée aussi par la répétition de l'image du 'détournement' à l'ouverture (12.4: debuerant fusos evoluisse) et à la fin de l'épître (12.211: versat), qui nous invite à lire le texte jusqu'au bout, comme dans le cas de Cydippe (21.111: perlege), ou de s'en apercevoir et d'en tenir compte, comme dans le cas d'Ariane (10.135: adspice mente). La lettre de Médée devient ars memoriae (12.1: memini; 12.1: ars) qui établit ses liens avec la figure en pierre de la lettre 10, comme le montre la répétition du terme vertere tout à la fin des deux épitres (10.149 et 12.211). ${ }^{52}$ Le dialogue nous permet de constater que la demande de l'héroïne délaissée de ne pas être oubliée (10.129: me quoque narrato sola tellure relictam) et son besoin d'être aperçue (10.135: adspice mente) seront satisfaits dans la lettre de Médée (12.212: Viderit ista deus), qui s'en souviendra (12.1: memini).

Contrairement à Médée, Ariane et Cydippe ne soulignent pas à l'ouverture de leur texte la notion de la mémoire, mais celle de l'oubli, en la reliant à l'art de la fraude (insidias). Cydippe insidiata $(21.2)^{53}$ reprend le jeu de polysémie que revêt l'ars de la parole, à première vue en réponse à Acontius, qui joue sur l'alternance des termes et leurs synonymes et qui définit à son gré l'art en tant que bonus dolus et la fraude en tant qu'écriture épistolaire (20.33-36: Si fraus huic facto nomen dicarque dolosus, / Si tamen est, quod ames, velle tenere, dolus. / En, iterum scribo mittoque rogantia verba, / Altera fraus haec est, quodque queraris habes). ${ }^{54} \mathrm{~A}$ la fin de la lettre

49 Sur cette traduction voir OLD s.v. oculus 1.

50 Voir OLD s.v. lumen 10.

51 Sur cette lecture voir OLD s.v. lumen 9.

52 Le terme apparaît à l'avant-dernier vers dans les deux textes.

53 Sur ce vers voir l'édition de Showerman $1984^{2}$.

54 Videau 2004: 7 suggère qu'on détermine si l'acte d'Acontius est un bonus dolus ou un malus dolus, mettant en évidence que dans le droit romain le plus ancien, tromper, par exemple, pour obtenir un meilleur prix d'un objet constitue un bonus dolus. 
21, l'emploi du terme artus (21.247: Iam satis invalidos calamo lassavimus artus,) suscite une identification paradoxale entre la figure de la femme et sa texture poétique, lorsqu'artus désignant les membres du corps et ars en tant que corpus textuel s'entrecroisent dans un synchronisme autant acoustique qu'optique; l'art de la parole paraît ainsi propre à séduire parce qu'il devient constitutif de l'autoportrait féminin. ${ }^{55}$

Le fruit, tout comme la double lettre, est porteur d'un message, d'un carmen (21.237-38: Hoc deus et vates, hoc et mea carmina dicunt. / A! desunt voto carmina nulla tuo), ${ }^{56}$ qui implique un charme, une parole d'essence incantatoire ou poétique, comme pour vates $(21.237) .{ }^{57}$ De fait, plus que les vers inscrits sur la pomme et donc la formule inventée par Acontius, et plus que les vers qui composent la lettre de Cydippe, carmen désigne la

Kenney 1970: 395 souligne l'invalidité selon les principes du droit romain des revendications fondées sur le dolus. Sur le terme dolus dans les lettres d'Acontius et $\mathrm{Cy}-$ dippe voir aussi Frechet 2004: 97-113.

55 Selon Gavoille 2004: 209, "[1]a pomme, tout à la fois cadeau érotique, mythique objet de tentation, et même symbole de la perte d'innocence, porte ici un message très particulier (littera), qui dans l'Art d'aimer fournit le modèle de la séduction par lettre." Les histoires de pommes font figure de symbole érotique étant souvent annonciatrices de mariage. Voir Viarre 1988: 772-73, qui mentionne également que Catulle déjà ouvrait la voie à Ovide en faisant précéder sa traduction de La boucle de Bérénice par un autre poème (65), où la fuite des souvenirs est comparée à celle d'une pomme s'échappant de la tunique d'une jeune fille qui l'a reçue en cadeau furtif et qui rougit d'un aveu involontaire.

56 Sur la signification du terme carmen voir Guittard 2007: 6 qui mentionne que "[l]e carmen est lié à toutes les formes de la vie juridique et religieuse de la cité et on le trouve associé à la transcription des prophéties ou des réponses rendues par les dieux aux questions que les hommes leur ont posées, dans un cadre officiel ou privé." Le critique ajoute également que "les formules qui transcrivent des prophéties, c'està-dire le langage des dieux devenu accessible et rendu intelligible aux hommes." Voir aussi Dangel 1997: 114. Sur le terme carmen par rapport à la poésie et à la magie voir Fulkerson 2002: 61-87.

57 Sur la signification du terme vates, désignant le poète lui-même et conservant sa valeur magico-religieuse, voir Foulon 1990: 66-79. Sur la notion de vates chez Ovide voir Newman 1967: 100 sqq. 
prophétie d'Apollon, ${ }^{58}$ qui dévoile le vrai ou qui construit une forme nouvelle de vérité, celle-ci étant, selon les critiques, une parole dotée d'efficacité pragmatique, ${ }^{59}$ comme pour poeta. Le titre de magnus poeta dans le texte de Cydippe (21.112), chargé d'hommage respectueux autant que d'ironie, permet de célébrer non seulement le stratagème du héros, mais aussi l'astuce de Callimaque; ${ }^{60}$ poète au sens étymologique, l'épistolier fictif a fait preuve de sa capacité à créer une réalité, par la seule force des mots. $^{61}$

La réalité qu'Acontius a créée se dévoile à partir de ses effets sur la santé de Cydippe: à l'heure de la rédaction, ses mains malades et privées de force refusent un plus long office (21.248: Et manus officium longius aegra negat). Les mains sont ici porteuses d'un symbolisme autant métapoétique que juridique: l'inefficacité de l'héroïne d'achever son texte révèle son incapacité à offrir sa main, au sens figuré et au sens propre, à celui qui est prétendument son futur époux, et donc d'effectuer un mariage cum manu. ${ }^{62}$ La personnification dans son texte du dieu du Mariage qui s'enfuit (21.159-60: Ter mihi iam veniens positas Hymenaeus ad aras / Fugit,) et la focalisation sur la main paresseuse (21.160-62: et a thalami limine terga dedit, / Vixque manu pigra totiens infusa resurgunt / Lumina,) privilégient ainsi de surcroît une lecture juridique du terme.

Le lexique du droit et de la manus en particulier domine également la lettre de Médée, qui, victime de la parole fallacieuse, d'un carmen frauduleux, rappelle que Jason, se remettant entre ses mains, se livra à elle en tant que propriété (12.73-74: Ius tibi et arbitrium nostrae fortuna salutis /

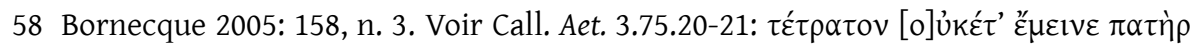
$\varepsilon$ c.... .......[ / Фоĩßov. [Le père n'attendit pas une quatrième épreuve; il fit voile vers Delphes, vers Phoibos.].

59 Gavoille 2004: 224.

60 Barchiesi 1993: 357.

61 Bopp 1966. La figure métapoétique d'Acontius a été soulignée à maintes reprises par les critiques. Voir en particulier Gavoille 2004: 225.

62 Selon le mariage sine manu, la femme peut rester liée à sa famille d'origine, tandis qu'avec le passage dans la manus (le mariage cum manu), elle est obligée de perdre ses liens avec sa famille. Même si c'est la femme qui veille sur sa fortune et administre ses propres biens, l'époux contrôle les actes; l'auctoritas du tuteur est nécessaire pour intenter des actions en justice, ou s'engager dans un contrat. Sur ce sujet voir Ducos 1996: 50 sqq. 
Tradidit inque tua est vitaque morsque manu). ${ }^{63}$ La riposte juridique de l'héroïne vis à vis de ce geste prend à l'heure de la rédaction la forme d'une actio d'asservissement non réalisée (12.157-58: Vix me continui quin sic lamiata capillos / Clamarem 'meus est' iniceremque manus), qui consiste en la manus iniectio, une action juridique selon laquelle le créancier pose sa main sur le débiteur. ${ }^{64}$ Le geste est, en effet, retenu; Médée se contient juste assez pour ne pas crier 'Il est à moi' en jetant la main sur le héros ${ }^{65}$. Cependant, en écrivant 'meus est' (158) elle fait agir son texte, qui parle à son insu, silencieusement - à la façon de Cydippe (21.1: sine murmure).

De manière similaire, Ariane étale sa main vers Thésée (10.40: iactactae manus; 10.146: tendo manus) et évoque le pactum (10.92: memini quae tibi pacta fui), dans une illustration ambiguë qui révèle à la fois une revendication de celui qui lui avait été promis et une offrande symbolique qui rappelle le fil offert dans le labyrinthe (10.104: fila dedi). Il devient néanmoins progressivement évident qu'Ariane et Médée ne partagent pas tout à fait le même raisonnement: Médée exige que Jason restitue son aide et ajoute que l'acquisition du héros a été bien méritée (12.102: Per meritum et 12.197: Te peto, quem merui), alors qu'Ariane précise qu'elle ne conjure pas Thésée au nom de ses bienfaits (10.141: Non te per meritum [...] adoro). En effet, la figure plastique d'Ariane n'a pas la posture animée de Médée; jeune, faible et isolée, elle rappelle la figure dévastée de la dernière 'écrivaine' du recueil, qui, malheureuse, les membres brûlant de fièvre, focalise ses derniers mots sur son état de santé et offre finalement,

63 En prononçant Effice me [...] tuum (82), le héros s'offre à Médée et joue ainsi le rôle d'un esclave qui appartient à son maître et qui est défini comme une res. Sur la définition du terme res voir Ducos 1996: 33 et suiv. qui précise qu'il s'agit d'un bien "sur lequel s'exerce pleinement le droit de propriété".

64 Sur la manus iniectio voir Ducos 1996: 184. Voir également Berger 1991, s.v. Legis actio per Manus Iniectionem: 'This legis actio was a form of a personal execution on the debtor for specific claims. Its name comes from a symbolical seizure on the debtor by the laying of a hand (manum inicere) upon him'. Voir également p. 577, sur la manus iniectio, la manus iniectio iudicati, la manus iniectio pro iudicato, et la manus iniectio pura.

65 Ducos 1996: 33-34 précise que "(l'esclave) peut être revendiqué en justice. Son maître possède sur lui une puissance absolue exprimée en général par le terme de potestas. Ce substantif, qui s'utilise pour désigner toute puissance reconnue par le droit, apparaît pour rendre manifeste le pouvoir du maître, qui est à la fois droit de coercition et pouvoir de vie et de mort." 
inconditionnellement, ses mains malades à Acontius (21.242: Doque libens victas in tua vota manus).

La manus se transforme ainsi de modus rhétorique en actio juridique qui fait appel à la revendication, qui proteste au mariage, qui rappelle l'offre, mais qui est aussi propice à une anamorphose de l'écriture. De fait, s'appuyant sur la référence aux mains fatiguées de l'écrivain, des spécialistes d'Ovide ont mis en exergue que la dernière des héroïnes cède éventuellement au stratagème de l'homme orateur. ${ }^{66}$ Néanmoins, une lecture intratextuelle favorise des interprétations différenciées. Dans la lettre 20, Acontius se réfère au simulacre de la pomme, et met en évidence la forme épigrammatique de l'inscription, afin d'imposer sa réalisation, notamment son union avec l'héroïne (20.239-42: Aurea ponetur mali felicis imago, / Causaque versiculis scripta duobus erit: / 'Effigie pomi testatur Acontius huius, / Quae fuerint in eo scripta, fuisse rata.'). ${ }^{67}$ Cydippe y répond, en se référant au simulacre non plus d'une pomme mais d'une autre lettre: elle crée donc l'illusion qu'un autre texte stratégique du héros aurait pu la faire encore survivre (21.239-40: Vnde tibi favor hic? nisi si nova forte reperta est. / Quae capiat magnos littera lecta deos;), précisant que le reste relève d'Acontius (21.245: cetera cura tua es). L'héroïne rétablit ainsi ses liens dialogiques autant avec le texte de Médée - qui explique qu'en ce qui concerne le reste, elle 'laisse faire au dieu' (12.212: Viderit ista deus) - qu'avec le texte d'Ariane, qui exprime son besoin d'être aperçue par l'esprit (10.135: adspice mente), afin d'être sauvée - par un dieu, notamment Dionysos. Plus encore, cette illusion scripturale génère une série d'allusions, en dépliant la menace de Médée et celle d'Ariane, ailleurs réalisées: d'une part le tissu empoisonné à être offert à Créuse (12.17980: Rideat et Tyrio iaceat sublimis in ostro. / Flebit et ardores vincet adusta meos) et d'autre part le voile noir qui conduira Égée à la mort (Catull. 64.199201: Vos nolite pati nostrum vanescere luctum, / Sed quali solam Theseus me

66 Selon Ziogas 2016: 229: 'Even within the fictional world the likeness of the apple is merely a fantasy, yet its realization depends on Acontius marrying Cydippe, a happy ending known to the reader, who can thus entertain the realization of the golden apple by indulging in Acontius' fantasy'. Voir aussi Barchiesi 1999: 53-67, qui a remarqué que, “[m]ince et fragile, consumée par la fatigue de l'écriture, Cydippe incarne un modèle de poétique callimaquéenne (XXI, 15-28)."

67 Sur l'association générique entre l'épigramme et les Héroïdes voir Casanova-Robin 2005: 131. 
mente reliquit, / Tali mente, deae, funestet seque suosque.»). Par cette menace réitérée en puissance, Cydippe encourage Acontius, le grand poète (21.112), à ne pas imiter Jason et Thésée, et à devenir le vates (21.237) qui l'aura sauvée.

En donnant suite à l'œuvre ovidienne par un nouveau texte et en privilégiant une lecture différenciée de la lettre 'féminine', Cydippe met potentiellement fin à cette 'généalogie littéraire' des femmes malheureuses. Bien que le regard de chacune des 'épistolières' soit différent, cette posture polyphonique devient un lien unissant les trois personnages. Ainsi, en plaidant sa cause jusqu'au bout (21.154: causa peracta mei est), Cydippe a 'déjà dit plus que ne doit dire une jeune fille' (21.245: plus hoc quoque virgine factum), parce qu'elle a déjà évoqué dans son épître les voyages souvenus par Médée et Ariane, les aperçus et les aspirations de ces héroïnes. En rejetant la rhétorique du silence féminin que représente l'intertexte callimaquéen, elle trouve finalement refuge dans le monde ovidien, où la femme voyageuse et exilée, placée en marge de la société, est évoquée et ainsi revue. Cydippe renvoie à Ariane, la puella abandonnée par excellence qui rompt ses liens avec son alter ego, l'ecphrasis catullienne, et à Médée, la femme criminelle, qui exploite la puissance du discours juridique pour défendre sa cause. Les jeux d'identifications et de distanciations entre la dernière héroïne et les deux femmes exilées permettent surtout à Ovide, peut-être déjà en exil, ${ }^{68}$ de demander d'être aussi aperçu par l'esprit (10.135: mente, donc lu 'jusqu'au bout': 21.154), lorsque la lettre demeure son seul moyen de réparation. En déclarant, à travers le texte de Cydippe, que 'le reste est ton affaire' (21.245: cetera cura tua est), le poète semble insinuer que l'avenir de l'auteur incombe à la réception de son œuvre. Le lecteur est ainsi appelé à voyager vers l'ailleurs des espace-temps radicalement autres, inattendus mais ingénieux, afin de faire encore survivre les Epistulae Heroidum, comme une poésie du seuil et une poésie du transitoire.

68 La date de la composition et celle de la publication du recueil ne peuvent pas être établies avec certitude, mais les lettres doubles sont plus récentes aue les lettres simples. Voir une discussion chez Fulkerson 2009: 78-80. Cf. Casanova-Robin 2007: 7. 


\section{BIBLIOGRAPHIE}

Aresi, L. 2017. Nel giardino di Pomona. Le "Metamorfosi" di Ovidio e l'invenzione di una mitologia in terra d'Italia. Heidelberg.

Armstrong, R. 2006. Cretan Women: Pasiphae, Ariadne, and Phaedra in Latin Poetry. Oxford.

Barbaud, T. 2006. Catulle: une vie poétique de l'indicible (Bibliothèque d'Etudes Classiques 47). Paris \& Leuven.

Barchiesi, A. 1993. 'Future Reflexive: Two modes of Allusion and Ovid's Heroides' HSPh 95, 333-65.

Barchiesi, A. 1999. 'Vers une histoire à rebours de l'élégie latine: les Héroïdes «doubles» (16-21)' in J. Fabre-Serris \& A. Deremetz (eds.) Elégie et épopée dans la poésie ovidienne (Héroïdes et Amours), en hommage à Simone Viarre. Villeneuve d'Ascq, 53-67.

Barchiesi, A. 2001. Speaking Volumes: Narrative and intertext in Ovid and other Latin poets. London.

Berger, A. 1991. Encyclopedic dictionary of Roman Law. Vol. 43. Philadelphia.

Beye, C.R. 1982. Epic and Romance in the Argonautica of Apollonius. Carbondale \& Edwardsville.

Bolton, M.C. 2009. 'Gendered Spaces in Ovid's Heroides' CW 102.3, 273-90.

Bopp, H. 1966. Inscia capta puella, Akontios und Kydippe bei Kallimacbos und bei Ovid. Diss. Mïnster.

Bordas, É. 2010. 'Sujet narrant: l'effet point de vue' L'Information grammaticale 124, 54-55.

Bornecque H. 2005 [1928]. Ovide, Héroïdes. Traduit par M. Prévost, 5e tirage de la $5^{\mathrm{e}}$ édition revue, corrigée et augmentée par D. Porte. Paris.

Burton, C. 1908. 'The Authorship and the Date of the Double Letters in Ovid's Heroides' HSPh 19, 121-55.

Cairns, D.L. 2002. 'The Meaning of the Veil in Ancient Greek Culture' in L. Llewellyn-Jones (ed.) Women's Dress in the Ancient Greek World. London \& Swansea, 73-93.

Casanova-Robin, H. 2005. 'Le simulacre et l'empreinte: poétique de l'image de l'autre dans les Héroïdes d'Ovide' BAGB 2, 117-34.

Casanova-Robin (ed.), H. 2007. Amor scribendi. Lectures des Hérö̈des d'Ovide. Grenoble. 
Casanova-Robin, H. 2007. 'Poétique du corps et représentation de soi dans les Hérö̈des d'Ovide' in H. Casanova-Robin (ed.) Amor scribendi. Lectures des Héroïdes d'Ovide, 53-79.

Casanova-Robin, H. 2008. 'Duel singulier dans les Héroïdes d'Ovide (XX et $\mathrm{XXI}$ ): symétries et divergences autour de l'herméneutique du signe' Euphrosyne 36, 71-85.

Clare, R.J. 1996. 'Catullus 64 and the Argonautica of Apollonius Rhodius: Allusion and Exemplarity' PCPhS 42, 60-88.

Conte, G.B. 1986. The Rhetoric of Imitation. Genre and Poetic Memory in Virgil and Other Latin Poets. Ithaca \& London.

Dangel, J. 1997. 'Le carmen latin: rhétorique, poétique et poésie’ Euphrosyne $25,113-31$.

Dangel, J. 2002. 'Catulle, carmen LXIV: mythe, amour et art poétique' in P. Defosse (ed.) Hommages à Carl Deroux. Vol. 1 (Latomus 266). Bruxelles, 127-41.

Davis, P. 2012. 'A Simple Girl'? Medea in Ovid Heroides 12', Ramus 41.1-2, 33-48.

DeBrohun, J.B. 1999. 'Ariadne and the Whirlwind of Fate: Figures of Confusion in Catullus 64. 149-57' CPh 94.4, 427-29.

Deroux, C. 1986. 'Some Remarks on the Handling of Ekphrasis in Catullus 64 ' in C. Deroux (ed.) Studies in Latin Literature and Roman History. Vol. 4. Brussels, 247-58.

Duban, J. 1980. 'Verbal Links and Imagistic Undercurrent in Catullus 64' Latomus 39, 777-802.

Ducos, M. 1984. Les Romains et la loi. Recherches sur les rapports de la philosophie grecque et de la tradition romaine à la fin de la République. Paris.

Ducos, M. 1996. Rome et le droit. Paris.

Duffalo, B. 2012. The Captor's Image: Greek Culture in Roman Ecphrasis. Oxford.

Elsner, J. 2007. Viewing Ariadne: from Ekphrasis to Wall paintings in the Roman World' CPh 102.1, 20-44.

Foulon, A. 1990. 'L'art poétique de Tibulle' REL 68, 66-79.

Fréchet, C. 2004. 'Quelques réflexions sur un vers des Héroïdes (Hér. XX, 123)' Dictynna 1, 97-113.

Fulkerson, L. 2002. '(Un)Sympathetic Magic: A Study in Heroides 13' AJPh 123.1, 61-87. 
Fulkerson, L. 2005. The Ovidian Heroine as Author. Cambridge.

Fulkerson, L. 2009. 'The Heroides: Female Elegy?' in P.E. Knox (ed.), A Companion to Ovid. Oxford, 78-89.

Gavoille, É. 2004. 'Acontius et Cydippe: Une pièce épistolaire (Ovide, Héroïdes XX-XXI)' in L. Nadjo \& É. Gavoille (eds.) Epistulae Antique 3. Actes $\mathrm{du}$ IIIe Colloque International L'Epistolaire Antique et ses Prolongements Européens, Université François - Rabelais, Tours, 25-27 septembre 2002. Louvain, 207-28.

Glare, P.G.W. (ed.) 1968. Oxford Latin Dictionary. Oxford. [OLD]

Guittard, C. 2007. Carmen et prophéties à Rome. Turnhout.

Goldhill, S. 2007. 'What is Ekphrasis for?' CPh 102.1, 1-19.

Hardie, Ph. 2002. Ovid's Poetics of Illusion. Cambridge.

Höschele, R. 2007. 'The Traveling Reader: Journeys Through Ancient Epigram Books' TAPhA 137.2, 333-69.

Jolivet, J.C. 2001. Allusion et Fiction Épistolaire dans les Héroïdes: Recherches sur l' intertextualité Ovidienne. Rome.

Jouteur, I. 2007. 'Le paysage marin des Héroïdes' in H. Casanova-Robin

(ed.) Amor Scribendi. Lectures des Héroïdes d'Ovide. Grenoble, 93-120.

Jouteur, I. 2009. 'Une lettre dans les coulisses de la tragédie (Hér. XII)' in I. Jouteur (ed.) La théâtralité de l'œuvre ovidienne. Paris, 67-88.

Kenney, E.J. 1970. 'Love and Legalism: Ovid, Heroides 20 and 21' Arion 9, 388-414.

Kinsey, T. E. 1965. 'Irony and Structure in Catullus 64' Latomus 24, 911-31. Knox, P.E. 1986. 'Ovid's Medea and the Authenticity of Heroides 12' HSPh 90, 207-23.

Knox, P.E. 1995. Ovid Heroides: Select Epistles. Cambridge.

Knox. P.E. 1998. 'Ariadne on the Rocks: Influences on Ovid, Her. 10' in P. E. Knox \& C. Foss (eds.) Style and Tradition: Studies in honour of Wendell Clausen. Stuttgart, 72-83.

Landolfi, L. 2000. Scribentis imago - Eroine ovidiane e lamento epistolare. Bologna.

Laird, A. 1993. 'Sounding out Ecphrasis: Art and Text in Catullus 64' JRS 83, 18-30.

Lindheim, S.H. 2003. Mail and Female: Epistolary Narrative and Desire in Ovid's Heroides. Madison. 
Lindsay, J. 1965. The clashing rocks: a Study of Early Greek Religion and Culture and the Origins of Drama. London.

McNally, S. 1985. 'Ariadne and Others: Images of Sleep in Greek and Early Roman Art' ClAnt 4.2, 152-92.

Michalopoulos, A.N. 2002. 'Ovid Heroides 10.1-4: Ariadne's MITOE' Mnemosyne 55.1, 95-97.

Michalopoulos, A.N. 2004. 'Fighting against a witch: the importance of magic in Hypsipyle's letter to Jason (Ov. Her. 6)' MHNH Revista Internacional de Investigación sobre Magia y Astrología Antiguas 4, 97-124.

Michalopoulos, A.N. 2014. Ovid Heroides 20-21: Acontius and Cydippe. Introduction, Text, Translation, Commentary. Athens 2014.

Michel, A. 1994². La Parole et la Beauté. Rhétorique et esthétique dans la tradition occidentale. Paris.

Newman, J.K. 1967. The concept of Vates in Augustan Poetry. Bruxelles.

Putnam, M.C.J. 1972. 'The Art of Catullus 64' in K. Quinn (ed.) Approaches to Catullus. Cambridge, 225-65.

Ramain, G. 1992. 'Sur la signification et la composition du poème 64' $R P h$, 46, 1922.

Rimell, V. 2006. Ovid's Lovers: Desire, Difference and the Poetic Imagination. Cambridge.

Scheid, J. \& J. Svenbro 1994. Le métier de Zeus: mythe du tissage et du tissu dans le monde gréco-romain. Paris.

Showerman, G. $1984^{2}$ [1914]. Ovid. Heroides. Amores. Revised by G.P. Goold. Cambridge, MA.

Spentzou, E. 2003. Readers and Writers in Ovid's Heroides: Transgressions of Genre and Gender. Oxford.

Stroh, W. 1991. 'Heroides Ovidianae cur epistulas scribant' in G. Papponetti (ed.) Ovidio poeta della memoria, atti del convegno internazionale di studi, Sulmona, 19-21 ottobre 1989. Roma, 201-44.

Theodorakopoulos, E. 2000. 'Catullus, 64: Footprints in the Labyrinth' in A. Sharrock \& H. Morales (eds.) Intratextuality: Greek and Roman Textual Relations, Oxford, 114-41.

Tola, E. 2005. La métamorphose poétique chez Ovide, Tristes et Pontiques. Le poème inépuisable. Paris.

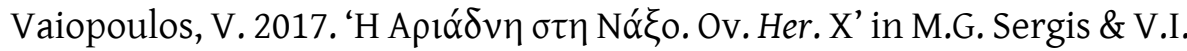

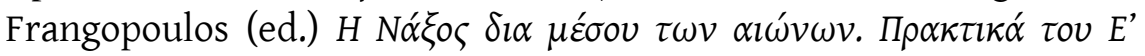




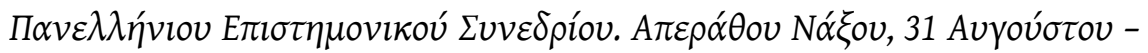
2 $\Sigma \varepsilon \pi \tau \varepsilon \mu \beta$ píov 2013, Athens \& Naxos, 27-43.

Verducci, F. 1985. Ovid's toyshop of the heart. Epistulae Heroidum. Princeton.

Viarre, S. 1988. 'Acontius et Cydippe ou la métamorphose d'un thème alexandrin dans les Héroides d'Ovide' Latomus 47, 772-84.

Videau, A. 2004. 'L'écriture juridique d'Ovide des élégies amoureuses (Amours et Héroïdes) aux Tristes de l'exil' Ars Scribendi 2, Le juridique, = http://ars-scribendi.ens-lyon.fr/article.php3?id_article=19.

Volk, K. 2003. 'Timeo simulacra deorum (Ovid, Heroides 10.95)' Mnemosyne 56.3, 348-53.

Waltz, R. 1945. 'Caractère, sens et composition du poème LXIV de Catulle' REL 23, 92-109.

Warden, J. 1998. 'Catullus 64: Structure and Meaning' CJ 93.4, 398-413. Ziogas, I. 2016. 'Love elegy and legal language in Ovid' in Ph. Mitsis \& I. Ziogas (eds.) Wordplay and powerplay in Latin poetry. Berlin \& Boston, 213-40.

Stella Alekou

University of Cyprus

alekou.stella@ucy.ac.cy 